\title{
Les violences envers les femmes dans la Caraïbe
}

Législations et données statistiques

Violence against women in the Caribbean: legislation and statistics

\section{Clara Palmiste et Nadine Lefaucheur}

\section{OpenEdition}

Journals

Édition électronique

URL : http://journals.openedition.org/plc/858

DOI : $10.4000 /$ plc. 858

ISSN : 2117-5209

Éditeur

L'Harmattan

Édition imprimée

Date de publication : 1 janvier 2012

Pagination : 21-42

ISBN : 978-2-296-55-856-4

ISSN : 1279-8657

Référence électronique

Clara Palmiste et Nadine Lefaucheur, "Les violences envers les femmes dans la Caraïbe », Pouvoirs dans la Caraïbe [En ligne], 17 | 2012, mis en ligne le 26 janvier 2012, consulté le 30 avril 2019. URL: http://journals.openedition.org/plc/858; DOI : 10.4000/plc.858 


\title{
LES VIOLENCES ENVERS LES FEMMES \\ DANS LA CARAÏBE \\ LÉGISLATIONS ET DONNÉES STATISTIQUES ${ }^{1}$
}

\author{
Clara PALMISTE \\ ATER \\ Membre associé du CRPLC \\ Université des Antilles et de la Guyane
}

Nadine LEFAUCHEUR

Sociologue

Membre associé du CRPLC

Université des Antilles et de la Guyane

Pour lutter contre les violences à l'égard des femmes, les états et institutions de la Caraïbe peuvent s'appuyer sur les trois traités majeurs qui ont fixé le cadre de la protection des femmes au niveau international ou régional: la Convention sur l'élimination de toutes les formes de discrimination à l'égard des femmes (CEDAW) adoptée en 1979 par l'Assemblée générale des Nations Unies; la Convention interaméricaine pour la prévention, la sanction et l'élimination de la violence contre la femme adoptée en 1994 à Belém do Para et la Déclaration et Programme d'actions de Beijing' ${ }^{2}$, adoptée lors de la quatrième conférence mondiale de l'ONU sur les femmes en 1995.

Les Etats signataires de la CEDAW s'engagent à tout mettre en œuvre pour éradiquer la violence à l'égard des femmes, en modifiant les pratiques juridiques ou coutumières qui encouragent la persistance ou la

${ }^{1}$ Cet article, qui exclut de son champ d'analyse les départements français d'Amérique (Guadeloupe, Guyane, Martinique), se fonde largement sur les informations rassemblées par :

- la Base de données du Secrétariat général de l'ONU sur la violence contre les femmes, créée en 2009 (un.org/ vawdatabase) ;

- le rapport rédigé par Jackie Sealy-Burke pour la Commission économique pour l'Amérique latine et la Caraïbe (ECLAC) et pour le Fonds de développement des Nations Unies pour les femmes (UNIFEM), Eliminating Gender-based Violence, Ensuring Equality, ECLAC/UNIFEM Regional Assessment of Actions to End Violence Against Women in the Caribbean, décembre 2003,

(http://www.eclac.org/publicaciones/xml/0/38620/lcarg764.pdf) ;

- Myriam Cottias. Rapport de l'enquête pilote sur les violences envers les femmes dans les DFA [Guadeloupe, Guyane, Martinique], CRPLC, 2001 ;

- Clara Palmiste. "Les violences conjugales : bilan des recherches et des plans d'actions dans la Caraïbe anglophone », Bulletin de la société d'histoire de la Guadeloupe, Gourbeyre, Archives départementales, n 157, 2010, p. 41-53.

${ }^{2}$ Le programme d'actions de Beijing renforce les dispositions de la CEDAW. 
tolérance des actes de violence. Les Nations Unies ne disposent cependant d'aucun moyen pour contraindre les Etats parties à mener à bien ces objectifs.

La plupart des états caribéens ont aujourd'hui signé cette convention $^{3}$, ainsi que la Convention interaméricaine de Belém do Pará ${ }^{4}$. Celle-ci définit la violence contre la femme comme «tout acte ou comportement fondé sur la condition féminine qui cause la mort, des torts ou des souffrances physiques, sexuelles ou psychiques à la femme, aussi bien dans sa vie publique que dans sa vie privée ».

C'est à propos des violences sexuelles que la mobilisation contre les violences faites aux femmes a commencé dans la Caraïbe, au tournant des années 1980-1990 - époque où seul Porto Rico y disposait d'une législation portant spécifiquement sur les violences domestiques - avec la Conférence sur «Les femmes, la violence et la loi », organisée en janvier 1990 par la CAFRA $^{5}$, pour mettre en relation les forces de police, les travailleurs sociaux, les juristes et les associations féminines afin de lutter plus spécifiquement contre le viol et les violences sexuelles.

Jusqu'en 1990, dans la plupart des états caribéens, les «crimes » de violence sexuelle et de violence domestique (non reconnue en tant que telle), relevaient du système pénal, auquel très peu de victimes avaient recours. Mais, à l'instigation de l'ECLAC, le Secrétariat du CARICOM ${ }^{6}$ a préparé, dans les années 1980, des modèles de législation sur la violence domestique, les infractions sexuelles et le harcèlement sexuel, qui ont servi de base aux réformes législatives intervenues à partir de 1991, les premiers états de la région à les avoir adoptées étant les Bahamas, Trinité-et-Tobago et le Belize, et le dernier étant le Suriname. Ces modèles, destinés à compléter les

\footnotetext{
${ }^{3}$ Avec des restrictions pour les Bahamas, Cuba, la Jamaïque et Trinité-et-Tobago.

${ }^{4}$ Celle-ci a été signée par Antigua-et-Barbuda, les Bahamas (avec des réserves), la Barbade, le Belize, la République Dominicaine, la Guyana, Saint-Kitts et Nevis, Sainte-Lucie, SaintVincent et les Grenadines, Trinité-et-Tobago, et ratifiée ultérieurement par la Dominique, la Grenade, Haïti et le Suriname.

${ }^{5}$ La Caribbean Association of Feminist Research and Action (Association caribéenne pour la recherche et l'action féministe) ou CAFRA, est un réseau féministe régional basé à Trinidad et regroupant des représentantes d'associations de femmes des états de la Caraïbe, dont plusieurs ont choisi de se consacrer à la lutte contre les violences faites aux femmes, comme l'association surinamienne Stop Violence Against Women Foundation, l'association trinidadienne Trinidad and Tobago Coalition Against Domestic Violence ou le Réseau féministe des Caraïbes et de l'Amérique latine contre la violence dans la famille et la violence sexuelle. La CAFRA a, en particulier, mené diverses campagnes de sensibilisation sur la violence conjugale et mis au point en 2000 un programme régional de formation destiné à la police (suivi par près de 5000 policiers, de 13 pays), aux officiers de justice et aux travailleurs sociaux.

${ }^{6}$ Caribbean Community and Common Market.
} 
dispositifs pénaux, visaient à protéger les victimes en prévenant la récidive par des ordonnances de protection, qui, relevant du droit civil, pouvaient être sollicitées par les conjoint(e)s ou ex-conjoint(e)s, les concubin(e)s ou ex-concubin(e)s, et les enfants de l'auteur de l'infraction (ou les enfants vivant ou ayant vécu sous son toit) - mais non par les visiting partners ${ }^{7}$. Cette exclusion des partenaires non cohabitants a été reprise par la plupart des états caribéens, mais certains pays, comme Trinité-et-Tobago ou la Dominique, ont modifié par la suite leur législation pour étendre la possibilité de solliciter des ordonnances de protection aux visiting partners (à la Dominique, toutefois, la relation doit durer depuis au moins un an) et/ou aux personnes ayant un enfant en commun avec l'auteur des violences. A la Dominique, des ordonnances de protection peuvent également être sollicitées par les parents ou les frères et sœurs de la victime, si celle-ci n'ose pas les solliciter elles-mêmes. Plusieurs états prévoient la possibilité que ces ordonnances de protection soient demandées par les services de police, voire par un procureur.

Une ordonnance de protection peut être sollicitée lorsqu'une personne a été victime, de la part de son conjoint ou partenaire, de violences physiques ou de menaces de violences physiques, ou de mental injury - la « cruauté mentale » étant différemment définie ou précisée selon les pays. À Trinité-et-Tobago, à la Dominique, à Grenade et à la Guyana, la violence économique (financial abuse) est également prise en compte lorsqu'elle vise à exercer un contrôle coercitif sur les ressources du conjoint ou partenaire, à exploiter ses ressources ou à limiter son accès aux ressources entraînant ainsi sa dépendance financière.

Les ordonnances de protection peuvent porter sur l'interdiction de «molester» ou de harceler la victime, sur l'exclusion de l'auteur des violences du logement familial et sur l'interdiction de s'en approcher, ainsi que des lieux que la victime fréquente habituellement (travail, écoles, etc.). Les législations les plus récentes (Trinité-et-Tobago, la Grenade, la Dominique, etc.) prévoient également la possibilité qu'elles obligent l'auteur des violences ou des menaces à payer certaines dépenses (loyers, remboursements d'emprunts, dépenses pour les enfants, dépenses médicales, frais de procès, etc.) et/ou à indemniser la victime. Mais ces ordonnances ne peuvent pas concerner l'attribution du «droit de garde» des enfants ni la fixation de pensions alimentaires. Dans certains pays, comme au Belize, la cour peut délivrer, pour les non-récidivistes, des ordres de réhabilitation les contraignant à suivre des programmes de rééducation. À Trinité-et-Tobago, les juges peuvent accepter des auteurs de violences des engagements de non-

\footnotetext{
${ }^{7}$ Terme utilisé par les anthropologues anglo-saxons pour désigner les personnes entretenant des relations sexuelles suivies, parfois pendant de nombreuses années, sans cohabitation et ayant éventuellement des enfants communs - l'un et/ou l'autre des partenaires (généralement l'homme, rarement la femme) pouvant avoir d'autres relations, avec ou sans cohabitation.
} 
récidive. Critiquées par les militant(e)s contre les violences conjugales, ces « secondes chances » données aux auteurs de violences ont été exclues des législations de Belize et de la Guyana en cas de violences physiques ou de récidive.

Le non-respect par les auteurs de violences des ordonnances émises à leur encontre - ou de leurs engagements à ne pas récidiver - constitue une infraction qui relève du droit pénal et peut entraîner la condamnation au paiement d'une amende ou à l'emprisonnement.

La plupart des états caribéens ont aujourd'hui promulgué une législation sur les violences sexuelles ${ }^{8}$, et, à l'exception notable de Cuba et Haïti ${ }^{9}$, sur la violence domestique ${ }^{10}$. Près de la moitié ont également reconnu

${ }^{8}$ Législations adoptées par Anguila (Domestic Violence law reform, 1996); Antigua-etBarbuda (1995) ; Aruba (2003) ; les Bahamas (1991) ; la Barbade (1992) ; le Belize (2000) ; Cuba (1979 et 1999, et, pour le harcèlement, 1997); la Dominique (1992 et 1998); la Guyana (1997 et 1998, pour le harcèlement, le témoignage par vidéo et le suivi des auteurs condamnés) ; Haïti (2005) ; la Jamaïque (1992, 2000, 2001, et, pour le harcèlement, 2007) ; Porto Rico (1998, amendement du code pénal limitant le délai dans lequel une victime de violence sexuelle peut porter plainte à cinq ans après les faits pour les adultes, et à cinq ans au-delà de la majorité, fixée à 21 ans, pour les mineures au moment des faits); la République Dominicaine (1997: violence et harcèlement); Sainte-Lucie (2005; 2000 pour le harcèlement dans l'emploi); le Suriname (2009); Trinité-et-Tobago (1986 et 2000, en particulier pour le harcèlement). Source: Base de données du Secrétariat général de l'ONU sur la violence contre les femmes.

${ }^{9}$ Haïti n'a remis aucun rapport à la CEDAW.

${ }^{10}$ Législations adoptées par Anguila (1996); Antigua-et-Barbuda (Domestic Violence Summary Proceedings - Act, 1999) ; les Bahamas (Sexual Offence and Domestic Violence Act, 1991 - loi complétée en 2007 par un Protection Orders Act, qui a élargi la définition des violences et étendu la protection de la loi aux couples non mariés), la Barbade (Domestic Violence - Protection Orders Act, 1992) ; le Belize (Domestic Violence Act, 1991 - amendé en 2000 - et 2007) ; les Bermudes (Domestic Violence - Protection Orders - Act, 1997) ; la Dominique (Protection Against Domestic Violence Bill, 1996 - amendé en 2002) ; la Grenade (Domestic Violence Act, 2001) ; la Guyana (Domestic Violence Act, 1996 - loi prenant en compte la violence économique - et 2008, pour le témoignage par vidéo et le suivi des auteurs condamnés) ; les Iles Caïmans (Summary Jurisdiction - Domestic Violence - Law, 1992) ; les Iles Vierges britanniques (Domestic Violence - Summary Proceedings - Act, 1992, Domestic Violence Act, 1995) ; la Jamaïque (Domestic Violence Act, 1994 - révisé en 2004 pour prendre en compte les couples non cohabitants et la violence économique, et permettre la demande d'ordonnances de protection par des tiers) ; Montserrat (Family - Protection Against Domestic Violence - Act, 1998) ; Porto Rico (1989) ; la République Dominicaine (1997 amendement du code pénal pour prendre en compte la violence domestique, le viol conjugal, le harcèlement sexuel et la violence sexuelle; modification de la constitution en 2000); Sainte-Lucie (Domestic Violence - Summary Proceedings - Act, 1994-1995) ; Saint-Kitts-etNevis (Domestic Violence Act, 2000 - loi amendée en 2005); Saint-Vincent-et-lesGrenadines (1984, Domestic Violence - Summary Proceedings - Act, 1995) ; le Suriname (2006 : adoption de protocoles relatifs aux plaintes pour violences conjugales -2009 : loi sur les violences conjugales); Trinité-et-Tobago (Domestic Violence Acts, 1991 et 1999). Sources : Base de données du Secrétariat général de l'ONU sur la violence contre les femmes et rapport ECLAC/UNIFEM, 2003). 
le viol conjugal ${ }^{11}$ et certains états ont adopté des mesures législatives contre le harcèlement sexuel ${ }^{12}$.

Tous les observateurs soulignent cependant la faible effectivité de ces lois et le recours limité aux ordonnances de protection de la part des victimes $^{13}$, ce que l'ECLAC impute à une «culture du découragement», stigmatisant les femmes qui traînent leur conjoint devant les tribunaux, mais s'appuyant aussi sur la peur des représailles et la dépendance affective et matérielle des femmes à l'égard de leur conjoint.

La question de l'importance des violences domestiques et sexuelles a, en effet, dans la Caraïbe comme ailleurs, été longtemps banalisée, considérée comme un fait culturel et relevant du domaine privé, et elle reste telle pour une grande part de l'opinion.

L'adoption générale de législations contre les violences sexuelles et domestiques ne découle toutefois pas seulement des sollicitations internationales. Elle répond aussi à une prise de conscience, encore très limitée et inégale ${ }^{14}$, des problèmes humains et sociaux posés par la violence dont souffrent la plupart des sociétés caribéennes - violence que l'on attribue généralement à leur histoire, marquée par la colonisation et l'esclavage, à leur situation économique difficile et à leur place, géographique et économique, dans les circuits de la drogue, mais aussi à un style d'éducation reposant largement sur les châtiments physiques et à un mode de socialisation fondant l'identité masculine sur la force physique, la supériorité

${ }^{11}$ C'est le cas d'Antigua-et-Barbuda (1995), des Antilles néerlandaises (1999 et 2003), des Bahamas (1991), de la Barbade (1992), du Belize (1999), de la République Dominicaine (1997), de Sainte-Lucie (2005) et du Suriname (2009).

${ }^{12}$ C'est notamment le cas du Belize (législation spécifique), des Bahamas (dans le cadre des infractions sexuelles), de la Guyana, de Sainte-Lucie et de Trinité-et-Tobago (dans le cadre des mesures antidiscriminatoires).

13 En 2000, 67 ordonnances ont ainsi été sollicitées à Antigua-et-Barbuda, 152 à SaintVincent-et-les-Grenadines, 275 à la Jamaïque, 472 à Sainte-Lucie et 6554 à Trinité-etTobago. Moins de la moitié des sollicitations aboutissent au prononcé d'une ordonnance (39\% à Trinité-et-Tobago en 1991-1994 et à Saint-Vincent-et-les-Grenadines en 1996-2001, $50 \%$ au Belize en 1993-1999 et $32 \%$ à la Jamaïque en 2000-2003). Il semble néanmoins que le recours aux ordonnances de protection soit plus important lorsque cette pratique est activement soutenue par les magistrats, comme à Antigua-et-Barbuda, ou dans des pays où, comme à Trinité-et-Tobago ou au Belize, l'adoption des législations protectrices a été précédée ou accompagnée d'une mobilisation importante des acteurs gouvernementaux ou de la société civile et de nombreux débats dans l'opinion publique (source: rapport ECLAC/UNIFEM, op. cit., 2003).

${ }^{14}$ Qu'ils émanent d'organismes internationaux publics ou privés, les rapports sur la Jamaïque soulignent ainsi que, en dépit de la gravité - et de l'aggravation - de la situation dans ce pays, les réponses des pouvoirs publics restent très insuffisantes, mal coordonnées et souffrant de l'absence d'analyses en termes de (socialisation) de genre (voir, en particulier, le rapport ECLAC/UNIFEM, 2003). 
des hommes sur les femmes et la «normalité »du recours à la violence comme expression de la sexualité masculine et de la virilitét ${ }^{15}$.

Cette violence reste cependant statistiquement mal documentée, particulièrement pour ce qui concerne les violences liées au genre : tous les observateurs reconnaissent l'absence, l'insuffisance ou la mauvaise qualité des données chiffrées sur la violence à l'égard des femmes dans la Caraïbe. $\mathrm{Si}$ des questions sur les violences conjugales et sexuelles ont été incluses dans certains pays, comme Haïti ou la République Dominicaine, dans les enquêtes portant sur la population ou sur la santé, aucune enquête spécifique de victimation ${ }^{16}$ n'a été réalisée en population générale. Pour approcher l'incidence des violences sexuelles et domestiques à l'égard des femmes, on ne peut se fonder que sur des données collectées par des institutions policières ou judiciaires, dans les pays qui ont adopté des protocoles d'enregistrement des données sur les violences domestiques ou de genre, comme la Barbade, le Belize, la Guyana et le Suriname. On dispose également de quelques recherches universitaires, dont les premières ont été réalisées à la Guyana à la fin des années $1980^{17}$, et de quelques études ou recensions effectuées par des associations féministes et/ou non gouvernementales, avec des financements locaux ou internationaux ${ }^{18}$.

\footnotetext{
15 «Violence is considered a regular or normal part of male sexual expressions and identity » Kamala Kempadoo. "Caribbean sexuality: mapping the field », Caribbean Review of Gender Studies, issue 3, 2009.

${ }^{16}$ Au contraire des statistiques institutionnelles, collectées par la police, la gendarmerie, la Justice, les organismes sociaux, etc., qui ne portent que sur les faits violents déclarés à ces institutions ou constatés par elles (ou sur les personnes qui ont été les auteurs et/ou les victimes de ces faits), les enquêtes dites de victimation interrogent des échantillons importants de personnes sélectionnées aléatoirement sur les faits dont elles ont pu être victimes, même si elles n'ont jamais porté plainte, voire n'en ont jamais parlé.

${ }^{17}$ George K. Danns \& Basmat Shiw Parsad. Domestic Violence and Marital Relationships in the Caribbean: A Guyana Case Study. Turkeyen, Women's Studies Unit, University of Guyana, 1989.

${ }^{18}$ Des rapports ou des études sur les violences à l'égard des femmes ont ainsi vu le jour, en particulier dans la Caraïbe anglophone, avec l'aide de l'UNIFEM ou dans le cadre du Commonwealth. Voir, en particulier :

- Ana Maria Brasileiro. Women Against Violence: Breaking the Silence. New York, UNIFEM, 1997 ;

- Commonwealth Secretariat. Violence Against Women. Curriculum Materials for Legal Studies, 1996 ;

- Roberta Clarke. Violence Against Women in the Caribbean: State and Non-State Responses. New-York, UNIFEM, 1998.
} 


\section{I. -LES GRANDES ANTILLES}

A Cuba (11 300000 hab.), où il n'y a pas de législation spécifique sur les violences exercées à l'encontre des femmes ${ }^{19}$, les autorités ont tendance à penser que «la violence contre les femmes n'est pas un problème » et que le harcèlement sexuel est «inexistant, en particulier dans les lieux professionnels et dans les institutions d'enseignement ${ }^{20}$. D'une enquête réalisée en 1992-1993 sur «la violence au quotidien» dans cinq provinces $^{21}$, portant sur 275 hommes et 225 femmes, il ressortait cependant que $58 \%$ des faits de violence «au quotidien » avaient lieu dans la sphère conjugale. Toutefois, les auteurs n'ayant postulé aucune différence de genre dans les manifestations de la violence, celles-ci n'avaient pas été comptabilisées par sexe et il n'était même pas possible de démêler qui, de l'époux ou de l'épouse, en était l'auteur ou la victime... ${ }^{22}$. Le rapporteur des Nations Unies estimait d'ailleurs, en 2000, que le manque cubain de statistiques sur les violences à l'égard des femmes - et même de statistiques sexuellement désagrégées quel que soit le domaine - restait un problème majeur, reconnu par la plupart de ses interlocuteurs.

En Haïti (9 600000 hab.), une enquête nationale sur l'évaluation de la violence à l'égard des femmes et des jeunes filles a été réalisée en 19941995, sous l'égide de l'UNICEF, par le Centre haïtien de recherche et d'actions pour la promotion féminine : 1705 femmes ont été interrogées en face à face dans les neuf départements du pays, sélectionnées parmi les habitantes des trois plus grandes communes ou d'autres communes ayant fait l'objet d'un tirage aléatoire ; $37 \%$ des femmes enquêtées ont déclaré être ou avoir été victimes de violences sexuelles (34\% de viols), $33 \%$ de violences physiques, tandis que des violences telles que «les injures, la privation arbitraire de liberté, le non-respect du devoir d'assistance, et l'asservissement » affectaient ou avaient affecté une femme sur quatre ; $36 \%$ des cas de violences rapportés étaient le fait du compagnon de la femme et les deux tiers des enquêtées ont déclaré n'avoir jamais dénoncé les faits dont elles avaient été victimes ${ }^{23}$.

\footnotetext{
${ }^{19}$ Mais, depuis 1999, l'existence d'un lien de famille entre l'agresseur et la victime y est considérée comme un facteur aggravant.

${ }^{20}$ Nations Unies, Conseil économique et social, Commission des Droits de l'Homme, 8 février 2000. Integration of the Human Rights of Women and the Gender Perspective. Violences Against Women. Report of the Special Rapporteur on Violence Against Women, its Causes and Consequences... Addendum : Report on the Mission to Cuba.

${ }^{21}$ Celeste Bermudez Savon \& Mirta Rodriguez Calderon. Violencia y leyes hogar adentro. La Havane, 1993.

${ }^{22}$ Myriam Cottias. Rapport de l'enquête pilote sur les violences envers les femmes dans les DFA (Guadeloupe, Guyane, Martinique).Schœlcher, CRPLC, 2001.

${ }^{23}$ Myriam Cottias, 2001. op. cit.
} 
L'enquête EMMUS-III (Enquête mortalité, morbidité et utilisation des services) réalisée en 2000 a inclus un module comprenant des questions qui fournissent des informations sur tout acte de violence commis par le mari ou par une autre personne sur les femmes depuis l'âge de 15 ans : $35 \%$ des femmes ont déclaré avoir subi des violences physiques depuis l'âge de quinze ans, dont $15 \%$ durant l'année précédant l'enquête. La proportion de femmes rapportant avoir subi une forme ou une autre de violence conjugale était de 30\% (18\% ayant fait état de violences physiques et $17 \%$ de violences sexuelles) ; $13 \%$ des hommes reconnaissaient d'ailleurs avoir déjà battu leur compagne.

Une enquête menée en $2004^{24}$ auprès de 880 jeunes filles et garçons scolarisés, âgés de 15 à 24 ans, révélait que, chez les jeunes filles sexuellement actives, $26 \%$ de celles qui avaient moins de 18 ans avaient déjà eu des rapports sexuels forcés et $74 \%$ de celles qui avaient plus de 18 ans avaient été victimes de violence sexuelle.

Un module sur les violences domestiques a de nouveau été intégré dans l'enquête EMMUS-IV de 2005. Des enquêtes qualitatives ont également été réalisées à l'initiative d'organisations non gouvernementales en 2002 et 2006, tandis qu'une enquête nationale sur la violence domestique et sexuelle «Une réponse à la violence faite aux femmes en Haïti » était réalisée en 2007.

En 2008, une ligne téléphonique spéciale a été créée pour les femmes victimes. L'année suivante a été créée une fiche nationale d'enregistrement des allégations de violences faites aux femmes et un centre public d'hébergement a été ouvert pour les femmes victimes; des centres d'hébergement ou des centres offrant des soins de santé gratuits aux femmes victimes ont également été mis en place par différentes ONG.

En Jamaïque (2 700000 hab.), la loi de 1994-1995 sur la violence domestique a reconnu celle-ci comme un crime. Les travaux préparatoires recommandaient de reconnaître le viol conjugal et le harcèlement sexuel, mais ces mesures attendent toujours d'être discutées par le Parlement ${ }^{25}$. Bien qu'envisagé depuis 1995 et prêt depuis 2007, le Sexual Offenses Bill en est en effet toujours, semble-t-il, à l'état d'avant-projet de loi. Le Comité sur l'élimination des discriminations à l'égard des femmes (CEDAW) a

\footnotetext{
${ }^{24}$ Dans le cadre du Projet PALIH d'appui à la lutte contre les ITSS/VIH/SIDA en Haïti : Ginette Noël \& Anne-Marie Thimoté. «Violence et prévention des ITSS. Expérience d'un projet de lutte en Haïti » p. 429-440, in Suzanne Arcand, Dominique Diamant, Sylvie Gravel. \& Elizabeth Harper (dir.). Violences faites aux femmes. Québec, Presses de l'Université du Québec, 2008.

${ }^{25}$ CEDAW. Cinquième Rapport périodique des Etats parties - Jamaïque. 2004. Sous le régime de la Common Law, le viol conjugal peut cependant parfois, mais très difficilement, être reconnu comme un crime.
} 
d'ailleurs attiré l'attention du gouvernement jamaïcain sur la lenteur des réformes législatives, ainsi que sur les niveaux élevés de violence domestique à l'égard des femmes, de viols, y compris conjugaux, et d'inceste.

Ces niveaux apparaissent en effet très élevés, voire extrêmes ${ }^{26}$. La violence conjugale apparait habituelle : en 1996, les femmes interviewées dans une communauté de Kingston et au tribunal familial de la ville pensaient ainsi que plus de sept hommes jamaïcains sur dix battaient leur femme (les hommes estimaient, quant à eux, que moins de six sur dix d'entre eux le faisaient $)^{27}$. Une enquête réalisée en 1997 dans cinq villes sous l'égide de la Banque Mondiale avait mis en évidence un lien étroit entre dépendance économique et violence familiale ${ }^{28}$. En 2004, le rapport du Comité CEDAW soulignait la fréquence des violences conjugales et des violences sexuelles à la Jamaïque : alors que, comme on le sait, une minorité seulement des victimes s'adressent à la police ou à la justice ${ }^{29}$, le nombre total de cas de violence domestique signalés s'était élevé en 2002 à 15 440, dont $71 \%$ s'étaient produits dans les régions rurales ; 1444 seulement, donc moins de $10 \%$, avaient donné lieu à une action en justice ${ }^{30}$. Un tiers des meurtres

26 «Les chiffres sont alarmants, ainsi que l'extrême cruauté des actes rapportés qui sont le signe d'une véritable déshumanisation des auteurs et d'une banalisation d'un phénomène qui semble échapper à tout contrôle. En moyenne, chaque jour 4 viols sont commis à la Jamaïque, et il ne s'agit là que du chiffre officiel. Les victimes sont non seulement des femmes, mais aussi un nombre croissant d'adolescentes et même d'enfants, environ trois quarts d'entre elles ont moins de 20 ans. Il s'agit souvent d'actes d'une extrême barbarie, viols en réunion assortis de violences physiques, de plus en plus souvent fatales ", écrivaient, par ailleurs, en février 2008, à propos de la Jamaïque, Steve et Stéphanie James, auteurs d'un projet de série documentaire sur les violences faites aux femmes dans la Caraïbe (Projet Le voile du silence, Shakti Productions).

Voir aussi :

- Amnesty International. Document - Jamaica, Sexual Violence Against Women and Girls in Jamaica : 'Just a little Sex'. 2006. (http://www.amnesty.org/en/library/asset/AMR38/002/2006) ;

- Nesha Z. Haniff. Male Violence against Women and Men in the Caribbean: the Case of Jamaica. Bridgetown, Women and Develoment Unit, 1995 ;

- Rapport ECLAC/UNIFEM, op. cit., 2003, p. 55-82.

${ }^{27}$ S. Lafont. The Emergence of an Afro-Caribbean Legal Tradition: Gender Relations and Family Courts in Kingston. Kingston, 1996.

${ }^{28}$ C. Moser, \& J. Holland. Urban Poverty and Violence in Jamaica. Washington D.C., The World Bank (World Bank Latin American and Caribbean Studies), 1997.

${ }^{29}$ Minorité estimée à $10 \%$ par Barbara Bailey, National Report on the Situation of Gendered Violence Against Women and Girls. Jamaica, UNDP, 1999.

${ }^{30}$ Base de données du Secrétariat général de l'ONU sur la violence contre les femmes, Jamaïque : "Justice System Data Regarding Complaints Filed under the Domestic Violence Act».

Le Woman Inc. Crisis Centre, association à but non lucratif créée en 1985, publie chaque année des statistiques concernant en particulier les cas de viol, d'inceste et de violence 
commis entre 1997 et 2002 ont été attribués à la violence domestique. Les services de police ont aussi enregistré 916 viols de femmes et 409 viols de mineures (de moins de 16 ans) en 2004 - et 655 viols de femmes entre janvier et octobre 2008 -, mais on estime que ces chiffres représentent moins du quart des viols réellement perpétrés ${ }^{31}$, surtout parmi les mineures. D'après le rapport d'Amnesty International sur la violence sexuelle en Jamaïque, $70 \%$ des agressions sexuelles visent des jeunes filles ${ }^{32}$. Selon une enquête menée auprès d'un échantillon aléatoire d'adolescentes âgées de 13 ou 14 ans, $17 \%$ d'entre elles avaient déjà connu au moins un viol ou une tentative de viol ${ }^{33}$. D'après une autre enquête, $20 \%$ des 15-19 ans auraient eu leurs premières relations sexuelles sous la contrainte ${ }^{34}$. Selon les statistiques criminelles, $86 \%$ des viols déclarés en 2002 et 2003 avaient été commis par un proche: membre de la famille, ami, partenaire ou connaissance ${ }^{35}$. Il semble aussi qu'une part non négligeable des rapports sexuels forcés ait eu lieu sous la menace d'armes.

En 2002, les féministes jamaïcaines ont publié un Manifeste pour la prévention de l'injustice sexuelle, signé par des ONG, des universitaires, des associations militantes ou religieuses. Le Premier Ministre a reconnu en 2003 que la violence domestique avait atteint un niveau «très préoccupant » en Jamaïque et une nouvelle loi sur la violence domestique a été adoptée en 2004, étendant le bénéfice de la loi de 1994-1995 aux femmes ayant des relations de type visiting partner. En reconnaissant l'égale capacité des deux conjoints à signer des contrats et à administrer les biens communs, la loi sur la Propriété familiale et les droits des conjoints, adoptée également en 2004, facilite la rupture des liens conjugaux par les femmes victimes de violences. Une campagne de sensibilisation a également été lancée en 2004 contre la violence à l'égard des femmes et contre l'inceste ${ }^{36}$.

domestique, cf. la base de données du Secrétariat général de l'ONU sur la violence contre les femmes.

${ }^{31} \mathrm{D}$ 'autant que la définition du viol est toujours limitée à la pénétration vaginale.

32 Just a little Sex... 2006, op. cit.

${ }^{33}$ Susan Patricia Walker \& al. Nutritional and Health Determinants of School Failure and Dropout in Adolescent Girls in Kingston, Jamaica. Washington, DC., International Centre for Research on Women, 1994.

${ }^{34}$ Reproductive Health Survey, National Family Planning Board, 2002.

${ }^{35}$ Cité par le rapport d'Amnesty International, 2006.

${ }^{36}$ Les peines encourues pour inceste sont toujours inférieures à celles encourues pour viol, bien qu'il soit envisagé, depuis 1995, de supprimer ces « circonstances atténuantes ». Selon le rapport d'Amnesty International (2006), si la grande majorité des participants à des focus groups sur le sujet se prononçaient nettement contre l'inceste, $2 \%$ d'entre eux estimaient quand même qu'il était «quelquefois acceptable » qu'un père ait des relations sexuelles avec ses filles. 
La Jamaïque a ratifié en 2005 la Convention interaméricaine de Belém do Para et le gouvernement a promis de financer des ONG travaillant dans le domaine de la lutte contre les violences à l'égard des femmes (tout en soulignant que les programmes d'ajustement structurel, la dette et la réduction des dépenses sociales ne permettront vraisemblablement pas d'atteindre les buts fixés...).

Une Conférence régionale a d'ailleurs été organisée à Kingston, Jamaïque, en novembre 2008 sur le thème de la lutte contre les violences sexuelles: «Caribbean Partnerships to End Sexual Violence in the Region", avec au programme des rapports des centres de crise ou associations des Bahamas, de la Barbade, du Belize, des Iles Caïmans, de la Dominique, de la Guyana, de la Jamaïque, de la République Dominicaine, de Sainte-Lucie, de Trinité-et-Tobago, et des Iles Turks et Caïcos.

A Porto Rico (3 900000 hab.), où la loi sur la violence domestique adoptée en 1989 exclut de son application les couples adultérins et les couples de même sexe, le problème de la violence, notamment domestique, se pose à grande échelle. Une enquête portant sur 5755 femmes âgées de 15 à 49 ans, réalisée en 1995, concluait que $13 \%$ d'entre elles avaient été victimes de violences au cours de leur vie conjugale ${ }^{37}$. En 1999, 21000 cas de violence domestique ont été enregistrés par le Département de police, qui a créé un Domestic Violence Program. On estime que la moitié des femmes tuées le sont par leur conjoint (ce qui a été le cas de 31 femmes en 2004) ${ }^{38}$.

En République Dominicaine (9 800000 hab.) ${ }^{39}$, le niveau élevé de la violence envers les femmes a été récemment jugé «alarmant» par le Procureur de la province de Santo Domingo. En 1999, une section du recensement avait été consacrée aux violences conjugales. Il est apparu qu'un tiers des femmes avaient déjà été victimes de violences physiques, le plus souvent de la part de leur mari ou ancien mari ; $13 \%$ des femmes avaient été victimes de violences physiques au cours des douze derniers mois; les plus touchées étaient les femmes séparées ou divorcées et les femmes habitant en zone rurale.

\footnotetext{
37 Arlette Gautier. «Les violences au sein de la famille », XXIV Congrès général de la population, 2003.

${ }^{38}$ ECLAC. Data on Violence Against Women in Latin America and Caribbean. Invited Paper. United Nations Statistics Division. Expert Group Meeting on Indicators to Measure Violence Against Women, Genève, 8-10 octobre 2007 (les données sur Porto Rico contenues dans ce document proviennent de l'Oficina de la Procuradora de las Mujeres).

${ }^{39}$ Ministry of Women's Affair/UNDP/UNFPA, 2000. Diagnostico de género en la reforma y modernizacion, 2000

Dominican Republic, Secrataria de Estado de la Mujer. Evaluacion de la Aplicacion de la Plataforma de Accion de Beijing en la Republica Dominicana, 1995-2000. Santo Domingo, 2000.
} 
Selon les statistiques officielles, entre 1990 et 2001, neuf femmes ont été assassinées par leur conjoint ou ex-conjoint en moyenne chaque mois et 133 femmes ont été tuées par leur conjoint ou ex-conjoint entre janvier et août $2008^{40}$. Selon une enquête démographique et sanitaire menée en 2002 auprès de 6807 femmes âgées de 15 à 49 ans, $27 \%$ avaient été victimes de violences conjugales au cours des douze mois précédant l'enquête ${ }^{41}$.

En 2007, le Congrès a décidé une campagne de « seize jours contre les violences à l'égard des femmes » et un plan décennal pour l'égalité et l'équité entre les genres a été adopté, incluant la lutte contre les violences à l'égard des femmes. Plusieurs associations dominicaines défendant les droits des femmes ont cependant publié en juin 2008 un rapport - Critical Path of Dominican Women Survivors of Gender Violence - insistant sur le fait que les femmes victimes de violences conjugales ou sexuelles qui portent plainte sont « re-victimisées » par le système judiciaire.

En 2010, la Constitution a été modifiée pour y inclure la prohibition de la traite, le droit à l'intégrité physique, psychologique et morale et le droit de vivre sans violence et des campagnes de prévention de la violence ont été lancées.

\section{II. - LES PETITES ANTILLES}

Dans les petites Antilles, en-dehors des îles les plus peuplées : les Bahamas, la Barbade et, surtout, Trinité-et-Tobago, peu d'enquêtes ont été faites et peu de statistiques ont été publiées sur les violences envers les femmes.

Les Bahamas (330000 hab.) ont le taux de viols (déclarés ou constatés) le plus élevé au monde : 135 cas en 2007 - année où a été adoptée une loi de protection des victimes de violences familiales (y compris pour les couples non mariés), de harcèlement et de violences sexuelles et de violences à l'égard des femmes. Plus de $16 \%$ des meurtres commis en 2007 l'ont été dans le cadre des violences domestiques ${ }^{42}$.

\footnotetext{
40 Amnesty International Report 2009. Information relevant to the Stop Violence Against Women Campaign. (http://www.amnestyinternational.be/doc/IMG/doc/SVAW_Annual_Report_Info_2009.doc)

${ }^{41}$ Arlette Gautier, 2003, op. cit.

${ }^{42}$ Crime, Violence, and Development : Trends, Costs, and Policy Options in the Caribbean, A Joint Report by the United Nations Office on Drugs and Crime and the Latin America and the Caribbean Region of the World Bank, 2009.
} 
A la Barbade (294 000 hab.), selon une enquête effectuée en 1990 auprès de 264 femmes âgées de 20 à 45 ans, $30 \%$ d'entre elles avaient subi des violences physiques et/ou sexuelles dans leur relation conjugale ${ }^{43}$. Selon les statistiques de la police, 1406 agressions avaient été enregistrées comme des cas de violence domestique en 2002, 498 en 2003 et 489 de janvier à septembre 2004. Un protocole d'enregistrement des déclarations de violences liées au sexe a été mis au point en 2008 et une commission chargée des questions liées aux violences à l'égard des femmes a été créée en 2009. Des campagnes de lutte contre les violences faites aux femmes ont été organisées dès 1997, dont la Campagne du Ruban Blanc ${ }^{44}$ en 2004, des interventions dans les établissements scolaires, des sessions de formation destinées aux policiers, aux travailleurs sociaux, aux professionnels de la santé, etc.

A Trinité-et-Tobago (1333000 hab.), la société souffre «d'une escalade exponentielle des phénomènes de violences, notamment liés à la drogue et à la guerre des gangs dans les quartiers sensibles. Il existe cependant un autre type de violence, beaucoup plus insidieux et menaçant, puisqu'il a lieu dans la sphère 'privée' du foyer, le lieu même censé servir de refuge et de protection contre les dangers du monde extérieur. Au cours de la dernière décennie, le problème de la violence domestique à Trinidad est devenu si alarmant qu'il (...) est arrivé au centre des débats (...). Après en avoir occulté ou sous-estimé l'importance pendant des années, les autorités gouvernementales reconnaissent enfin qu'il s'agit d'un problème majeur », écrivent les auteurs du projet de série documentaire sur les violences faites aux femmes dans six pays de la Caraïbe, Voile du silence, qui ont choisi de centrer sur les violences domestiques l'épisode tourné à Trinidad ${ }^{45}$.

En 1991, la loi trinidadienne sur les violences domestiques les a officiellement définies comme un crime. Un peu plus de 2000 demandes d'ordonnances de protection, émanant surtout de femmes, ont été ainsi adressées en moyenne chaque année aux tribunaux entre 1991 et $1994^{46}$. Plus de 3000 par an l'ont été en 1999 et 2000. Entre 1995 et 1999, 80 femmes et

\footnotetext{
${ }^{43}$ Arlette Gautier, 2003, op. cit.

${ }^{44}$ Ruban porté par les garçons et les hommes en signe d'engagement à ne jamais commettre de violences liées au genre et à ne jamais rester silencieux devant de tels actes.

45 Steve et Stéphanie James. Le voile du silence, projet de série documentaire sur les violences faites aux femmes dans la Caraïbe. Shakti Productions, 2008. p. 21.

${ }^{46}$ Mary Creque. A Study of the Incidence of Domestic Violence in Trinidad and Tobago from 1991-1993, Trinidad and Tobago Coalition Against Domestic Violence, 1995 ;

Charmaine Crawford. The Intensification of Violence Against Women in Trinidad and Tobago During the Period of Structural Adjustment. Major Research Paper for the Graduate Program in Women's Studies, 1996.
} 
32 enfants sont décédés, victimes de violences domestiques ${ }^{47}$. La ligne téléphonique contre la violence domestique mise en place par le Ministère de la culture et de l'équité entre les sexes a reçu, en 1998, 2611 appels, dont $84 \%$ provenant de femmes. Celles-ci appartenaient le plus souvent à la tranche d'âge 26-35 ans, $70 \%$ étaient mariées légalement ou coutumièrement, $17 \%$ célibataires, $3 \%$ divorcées et $10 \%$ séparées.

L'enquête sur les violences au sein de la famille réalisée en 2000 par Joan Rawlins ${ }^{48}$, auprès de 200 personnes issues des deux principaux groupes ethniques, portait sur l'attitude et l'expérience de la population face aux violences conjugales, ainsi que sur les raisons de celles-ci : $16 \%$ des personnes enquêtées ont affirmé avoir subi des violences physiques et psychologiques, plus des trois quarts d'entre elles étaient des femmes. Parmi celles qui ont porté plainte, une sur deux a déclaré avoir subi des violences physiques, lesquelles, dans quatre cas sur cinq, ont nécessité des soins médicaux. Les maris étaient les auteurs principaux de cette violence et il n'y avait pas de différences en termes de comportements violents entre les AfroTrinidadiens et les Indo-Trinidadiens. Selon Steve et Stéphanie James, il est pourtant, «à tort ou à raison, communément admis que les violences se manifestent de manière distincte selon le groupe religieux au sein duquel elles sont perpétrées. On dit par exemple que les musulmans battent sauvagement leurs femmes, alors que les hindous ont la réputation de tuer les leurs avant de se suicider. Chez les catholiques, on utilise subtilement la Bible pour remettre la femme à sa place, la contrôler, la museler. Quoiqu'il en soit, au-delà de ces subtiles nuances culturelles, reste le thème commun sous-jacent de la domination des femmes par la force. Une analyse plus approfondie révèle d'autres similitudes entre ces différents groupes, comme les disparités dans le mode de socialisation des filles et des garçons, l'éducation à deux vitesses, et les réticences des leaders religieux à reconnaître l'existence de violences domestiques dans leurs communautés ${ }^{49}$. Les résultats de l'étude de Joan Rawlins suggéraient également que la violence dans le foyer durant l'enfance, l'alcool ou l'usage de drogues prédisposaient à ces violences.

Devant l'ampleur du phénomène, des actions ont été entreprises par des organismes gouvernementaux ou des ONG. La Division de l'équité entre les sexes organise ainsi des interventions dans les écoles et élabore des brochures d'information ( $\mathrm{La}$ violence dans les foyers: ce qu'il faut savoir »; «Guérison, un journal d'un survivant d'abus sexuel »; «Le droit à la liberté : un journal pour les survivants de violence conjugale »). L'ONG Coalition de Trinité-et-Tobago contre la violence domestique met

${ }^{47}$ CEDAW, Rapport initial des Etats parties - Trinité-et-Tobago, 2001.

48 Joan M. Rawlins. Domestic Violence in Trinidad: a Family and Health Problem", Caribbean Journal of Criminology and Social Psychology, vol. 5, $\mathrm{n}^{\circ} 1$ \& 2, 2000.

${ }^{49}$ Steve \& Stéphanie James, op. cit., 2008. 
l'accent sur les activités de prévention et la réinsertion sociale des femmes victimes de violence. Des associations masculines militent également pour l'élimination de la violence à l'égard des femmes. Entre 1996 et 1998, une centaine de policiers, le personnel des abris et centres de crise, des volontaires, des membres d'ONG, du personnel du Ministère du développement social et communautaire et des responsables de diverses communautés du pays ont participé à divers programmes de formation sur l'équité entre les sexes. Le programme de formation mis au point par la CAFRA a été la première tentative pour introduire une formation systématique sur le sexe et la violence dans les programmes de formation des policiers de Trinité-et-Tobago. En 2000, la CAFRA a également organisé, à l'UNIFEM, pour les magistrats, une table ronde sur les droits de l'homme et la discrimination à l'égard des femmes. Mais la base de données du Secrétariat général des Nations Unies sur les violences à l'égard des femmes indique qu'aucune information n'a été fournie par le gouvernement de Trinité-et-Tobago sur les travaux de recherche, les informations statistiques et les mesures d'accompagnement prises dans ce domaine.

Parmi les autres Petites Antilles, si Antigua-et-Barbuda (85000 hab.) publie quelques statistiques sur les cas de violences domestiques (Directorate of Gender Affairs), la base de données de l'ONU n'indique «aucune information disponible » concernant les travaux de recherche, les données statistiques et les mesures d'accompagnement pour la Grenade (106 000 hab.), Saint-Kitts-et-Nevis (50 000 hab.), Sainte-Lucie (165000 hab.), Saint-Vincent-et-les-Grenadines (120000 hab.). À notre connaissance, c'est seulement dans les Iles Vierges Britanniques et en Dominique que des enquêtes ont été effectuées sur les violences envers les femmes.

Dans les Iles Vierges britanniques (106 000 hab.), un rapport sur la condition des femmes avance que plus d'un quart des femmes interviewées auraient subi des violences conjugales. Cependant, nombre d'entre elles n'avaient jamais porté plainte, car elles considéraient que la police ne voudrait pas ou ne pourrait pas les protéger des violences une fois rentrées à la maison. La honte et le refus de criminaliser le partenaire violent constituaient également des freins au dépôt de plainte. $88 \%$ des femmes victimes de violences physiques disaient ne pas quitter leur partenaire violent par peur des représailles ; 78,2 \% disaient rester pour des raisons financières et $7 \%$ pour des raisons familiales ${ }^{50}$.

En Dominique (67 000 hab.) - qui a ratifié la convention CEDAW dès 1981 - trois enquêtes sur ce sujet avaient été menées en 1987, 1991 et

${ }^{50}$ The Development Planning Unit. The Status of Women in the British Virgin Islands, 1993. Voir aussi : Nesha Z. Haniff. A Study of Domestic Violence in the British Virgin Islands. "When the Women are Incapacitated, the Nation is Incapacited", 1998. 
1994, avant même la conférence de Beijing. La plus importante, celle de 1991, avait porté sur 1912 femmes, mariées, vivant maritalement ou chef de famille. Si cette enquête portait principalement sur «le statut de la femme», une partie des questions touchait aux violences. Les résultats indiquaient les taux de prévalence suivants: violences physiques sur un membre de la famille : $15 \%$; coups et blessures sur une femme : $13,9 \%$; maltraitances sur un enfant : $8,6 \%$; abus sexuels sur une femme : $6,3 \%^{51}$. Le Conseil national des femmes de la Dominique développe des activités de prévention et d'accueil (et, éventuellement, d'hébergement) des victimes ${ }^{52}$.

\section{III. - LA CARAÏBE CONTINENTALE}

Les trois états de la Caraïbe continentale, Belize, Guyana et Suriname, publient des statistiques administratives sur les violences exercées envers les femmes et des enquêtes y ont été effectuées sur le sujet par des universitaires, des associations ou des organismes publics.

Le Belize (288 000 hab.) publie chaque année depuis 2000 un rapport sur les violences domestiques, à partir d'informations collectées par les hôpitaux, les centres de santé, la police et le Women's Department. Il en ressort que les femmes y sont huit fois plus souvent victimes de violences conjugales que les hommes et que près de $90 \%$ de tous les cas enregistrés concernent des femmes âgées de 15 à 44 ans. La plupart ont un niveau scolaire primaire et sont actives, surtout dans les emplois de service domestique. Le nombre de cas collectés augmente chaque année d'environ $10 \%$; les premières années, ils concernaient surtout le groupe Créole, tandis que, depuis 2003, ils concernent principalement le groupe Métis, sans que cette évolution ait trouvé une explication. Plus de la moitié des cas sont des récidives et $70 \%$ se sont produits en zone urbaine - les travailleurs sociaux imputent aux croyances et aux traditions culturelles le fait que les districts du sud, où, selon eux, les violences domestiques sont courantes, mais non dénoncées, ne comptent que pour $3 \%$ des cas collectés. Les expertes du Comité CEDAW s'interrogeaient d'ailleurs en 2007 sur «l'efficacité de la nouvelle loi sur la violence domestique, qui est un problème qui continue d'affecter gravement la vie quotidienne des foyers à Belize, notamment dans les zones rurales du pays $»^{53}$.

\footnotetext{
${ }^{51}$ Myriam Cottias, op. cit, 2001.

${ }^{52}$ The Dominica National Council of Women (DNCW). Practical Ways to Prevent Domestic Violence. We are Family, 2000.

Voir aussi : rapport ECLAC/UNIFEM, op. cit. 2003, p. 43-55.

${ }^{53}$ Nations Unies, Comité pour l'élimination de la discrimination à l'égard des femmes. «Le rapport du Belize : Le Comité engage Belize à se doter d'un mécanisme national adapté au défi de la parité », juillet 2007. (http://www.aidh.org/Femme/Comite_FE/39-belize.htm)
} 
Une recherche menée en 1998 par l'Organisation du Belize pour les femmes et le développement ${ }^{54}$ suggérait une corrélation entre la violence conjugale et des facteurs tels que l'alcool, les drogues et le chômage. Les interviewées avaient ainsi identifié l'alcool (72\%), les drogues $(66 \%)$, le chômage (49 \%), la jalousie (65\%) et les relations extra-conjugales (61 \%) comme les principaux facteurs entraînant la violence conjugale. Il semble d'ailleurs que près d'un tiers des auteurs de violences conjugales étaient sous l'influence de l'alcool et plus d'un sur dix sous celle de stupéfiants.

Depuis 1999, des sections chargées de combattre la violence dans les foyers ont été créées dans les principales unités de police de Belize et plus de trois cents policiers ont reçu une formation spéciale. En 2003, la lutte contre les violences à l'égard des femmes a été incluse dans l'ensemble des politiques publiques. En 2005, un comité gouvernemental (Domestic Violence Task Force) a été constitué pour étudier l'ampleur et la nature de ce problème, ainsi que l'effectivité de la législation sur la violence familiale, et le Women's Department a organisé des ateliers de «conscientisation ». En 2007, s'est déroulée une campagne nationale de seize jours contre les violences à l'égard des femmes. Un plan d'action contre les violences à l'égard des femmes a été adopté pour les années 2008-2009, suivi d'un nouveau plan d'action contre les violences liées au genre pour les années 2010-2013.

C'est dans la République coopérative de la Guyana (738 000 hab.) que fut menée l'une des premières études sur les violences conjugales dans la Caraïbe. Effectuée en 1988-1989 par George K. Danns et Basmat Shiw Parsad $^{55}$, elle concluait que la violence conjugale était récurrente dans les relations maritales en Guyana, que les femmes ayant des enfants y étaient plus exposées que celles qui n'en avaient pas et que plus les enfants étaient nombreux et plus fréquents étaient les actes de violence. Danns et Shiw Parsad examinèrent également le lien entre la violence subie par les hommes durant leur enfance (en tant que victimes et/ou en tant que témoins) et leur usage de la violence envers les femmes une fois devenus adultes, dans les deux principaux groupes ethniques de la Guyana. Les résultats de l'enquête montrèrent que les Afro-guyanais battus durant leur enfance ou qui avaient assisté à des scènes de violence conjugale ont tous frappé leur compagne à l'âge adulte et que ceux qui avaient été battus fréquemment durant l'enfance étaient les plus violents devenus adultes. Dans cette même communauté,

${ }^{54}$ J. Iyo. Research Summary: a Study on Domestic Violence in the Belize District, Belize, Organization for Women and Development, 1999.

${ }^{55}$ Georges K. Danns \& Basmat Shiw Parsad. Domestic Violence and Marital Relationship in the Caribbean: A Guyana Case Study. Women's Studies Unit, University of Guyana, 1989.

Voir également : Gaietry Pargass \& Roberta Clarke. «Violence against women: a Human right issue post Beijing five year review », p. 42-43, in Gender Equality in the Caribbean. Reality or Illusion, 1998. 
deux tiers des hommes qui n'avaient pas subi ou été témoins de violences durant leur enfance frappaient également leur compagne. Les résultats étaient sensiblement différents dans la communauté indo-guyanaise : si la quasi-totalité $(97 \%)$ des hommes battus dans leur enfance et la grande majorité $(71 \%)$ des hommes ayant assisté à des scènes de violence conjugale, ainsi que les deux tiers de ceux qui n'avaient pas été eux-mêmes victimes durant leur enfance, battaient leur femme, les quatre cinquièmes de ceux qui n'avaient été ni témoins ni victimes ne la frappaient jamais - ou très rarement. Il ressortait donc de cette étude que la consommation d'alcool et les violences subies dans l'enfance ne suffisaient pas à expliquer le comportement violent des hommes guyanais envers leur compagne.

L'étude menée dix ans plus tard sur la violence domestique à la Guyana par le Red Thread, organisation féministe multi-raciale, différait de la précédente par la méthodologie utilisée et par les objectifs à atteindre ${ }^{56}$. Le but de cette recherche était en effet d'analyser comment les femmes percevaient la violence conjugale, son ampleur et la nature des violences. Sur les 360 femmes de la capitale (Georgetown) interviewées, $77 \%$ pensaient que la violence familiale était très répandue à la Guyana ; $83 \%$ identifiaient la violence physique comme étant de la violence conjugale, mais $50 \%$ considéraient également la violence verbale comme de la violence conjugale ; $32 \%$ ont déclaré avoir été battues durant l'enfance (84\% affirmaient avoir reçu des gifles, mais ne considéraient pas cela comme de la violence). Si huit femmes sur dix affirmaient que les violences physiques dans l'enfance avaient été commises par leur mère ou par une parente, les abus sexuels avaient été exclusivement commis par des hommes de la famille.

Les deux tiers des interviewées vivaient en couple: parmi elles, $28 \%$ avaient subi des violences physiques ; $26 \%$ des violences verbales et $13 \%$ des atteintes sexuelles. L'étude montra également que $79 \%$ des femmes victimes n'avaient pas porté plainte: $40 \%$ d'entre elles étaient pourtant allées voir la police ; $34 \%$ s'étaient confiées à un parent, $5 \%$ à un voisin, $3 \%$ à un prêtre et $3 \%$ à un conseiller. Et $20 \%$ affirmaient que la violence subie avait diminué après qu'elles eurent demandé de l'aide. Il s'est avéré que $68 \%$ des femmes ne connaissaient pas les lois qui condamnaient la violence conjugale, ni les organisations qui pouvaient les aider. Pour sept sur dix des $55 \%$ de femmes qui avaient subi des violences dans une précédente relation, la violence avait cessé parce que la relation avait pris fin, et non à cause du changement d'attitude du partenaire.

\footnotetext{
${ }^{56}$ Linda Peake. "Feminist and quantitative? Measuring the extent of domestic violence in Georgetown, Guyana", Treballs de la Societat Catalana de Geografia, n 66, 2009, p. 133148 ;

Voir aussi Clara Palmiste, op. cit.
} 
Une loi contre la violence domestique avait été adoptée en 1996. Mais c'est surtout depuis 2008 que le gouvernement de la Guyana a multiplié les initiatives et inauguré, avec la campagne «Break the Cycle, Take Control », une vaste politique nationale de lutte contre la violence domestique et sexuelle: Plan 2008-2013 de lutte contre la violence domestique et inclusion de la lutte contre la violence domestique dans le Plan stratégique de santé 2008-2012 ; création en 2009 d'un Comité national de surveillance de la violence domestique et en 2010 d'un Comité national pour la prévention des violences sexuelles et d'une commission pour l'égalité des genres; adoption en 2010 de plusieurs protocoles relatifs aux violences sexuelles et domestiques destinés aux magistrats, à la police et aux travailleurs sociaux ou conseillers conjugaux; formations destinées aux principaux intervenants impliqués dans la lutte contre les violences domestiques, et en particulier aux responsables religieux et communautaires; stages destinés aux auteurs d'agressions domestiques et sexuelles; ouverture de lignes téléphoniques spéciales, de refuges et de facilités pour l'obtention de micro-crédits par les victimes de violences domestique; plan stratégique de coordination avec les organisations guyanaises non gouvernementales (The Red Thread Women's Development Organisation, Help and Shelter et le Women's Affairs Bureau), les institutions régionales et internationales, la police, les magistrats, les avocats, les travailleurs sociaux, etc.

Au Suriname (458 000 hab. $)^{57}$, dernier pays de la Caraïbe à avoir adopté, en 2009, une législation sur les violences domestiques, la police publie, depuis 2006, des statistiques trimestrielles sur les violences conjugales déclarées (au total, 1181 cas en 2006 et 1165 cas en 2007). En 2006, l'enquête MICS (Multiple Indicator Cluster Survey), réalisée par le Bureau de statistiques, sous l'égide du Ministère des Affaires sociales et du logement et de l'Unicef, a porté sur un échantillon représentatif de 5746 ménages et comporté un module sur les violences familiales subies par les enfants et les femmes âgées de 15 à 49 ans : 13,2\% des femmes de cette tranche d'âge ont répondu qu'un homme avait le droit de battre sa femme ou sa compagne pour diverses raisons, en particulier si elles négligeaient leurs enfants. La proportion atteignait $24,4 \%$ pour les femmes parlant les langues des Noirs «marrons», 19,4\% pour celles qui parlaient des langues indigènes et $18,6 \%$ pour celles qui parlaient le Hindi surinamien.

Des formations spéciales sur la violence domestique sont dispensées à la police depuis 1997. En 2007, année où la police a enregistré 1245 plaintes pour violences conjugales, la journée internationale des femmes a été célébrée avec le mot d'ordre «En finir avec l'impunité pour la violence contre les femmes et les enfants ». En 2008, un comité directeur national sur

${ }^{57}$ Voir le rapport ECLAC/UNIFEM, 2003, op. cit., p. 82-102. 
la violence domestique a été constitué et un coordinateur spécial de la police nommé ; des émissions spéciales de radio et de télévision ont été diffusées. Une loi contre la violence domestique a été promulguée en 2009.

Ce panorama des données statistiques sur les violences à l'égard des femmes dans les états de la Caraïbe montre que ces données sont le plus souvent rares, manquantes ou partielles, et, lorsqu'elles existent, d'origine administrative et non standardisées. Des propositions de standardisation du recueil des données sur les violences conjugales sont cependant à l'étude ${ }^{58}$ en particulier, pour ce qui concerne l'Amérique latine et la Caraïbe, dans le cadre de la Commission économique des Nations Unies pour l'Amérique latine et la Caraïbe (ECLAC), qui a tenu ou participé à plusieurs réunions sur le sujet ${ }^{59}$.

Mais, si partielles et insuffisantes soient-elles, ces données permettent d'entrevoir l'ampleur et la fréquence des violences subies par les femmes dans la Caraïbe.

\footnotetext{
${ }^{58}$ Diane Almeras, Rosa Bravo, Vivian Milosavljedic, Sonia Montano, Maria Nieves Rico. Violence Against Women in Couples: Latin America and the Caribbean. A Proposal for Measuring its Incidence and Trends. ECLAC, série Mujer y desarollo, $n^{\circ}$ 40, 2004 ;

ECLAC. «Data on violence against women in Latin America and Caribbean. Invited Paper », Genève, octobre 2007 ;

Rapport des Amis du Président de la Commission de statistique de l'ONU sur les indicateurs de la violence à l'égard des femmes, Conseil économique et social des Nations Unies, décembre 2008 .

${ }^{59}$ Réunion internationale sur les statistiques et indicateurs de mesure de l'incidence et des tendances dans les violences envers les femmes en Amérique latine et dans la Caraïbe, La Paz, novembre 2001 ;

United Nations, Women and Development Unit, Inter-Agency Project «Making Gender Indicators available for Policy-making », Santiago du Chili, octobre 2004 ;

Réunion du Groupe d'experts des Nations Unies sur les indicateurs de mesure de la violence à l'égard des femmes, Genève, octobre 2007.
} 


\title{
RÉSUMÉ
}

Clara Palmiste et Nadine Lefaucheur. Les violences envers les femmes dans la Caraïbe : législations et données statistiques

Jusqu'au tournant des années 1980-1990, les crimes de violence sexuelle et de violence domestique (non reconnue alors comme telle) relevaient dans la plupart des états de la Caraïbe du seul système pénal, auquel peu de victimes avaient recours. Pour lutter contre les violences à l'égard des femmes, ces états peuvent aujourd'hui s'appuyer sur la Convention CEDAW de l'ONU (1979), sur la convention interaméricaine de Belém do Para (1994) et sur le programme d'actions adopté à Pékin en 1995. Depuis 1991, la plupart d'entre eux ont adopté des législations civiles sur la violence domestique et sexuelle, inspirées des modèles préparés par le secrétariat du CARICOM, qui complètent les dispositifs pénaux par des ordonnances de protection visant à prévenir la récidive. Les observateurs soulignent cependant la faible effectivité de ces mesures. Limitées, parcellaires et peu standardisées, les données statistiques permettent cependant d'entrevoir l'ampleur et la fréquence des violences à l'égard des femmes dans la Caraïbe.

\begin{abstract}
Clara Palmiste and Nadine Lefaucheur. Violence against women in the Caribbean: legislation and statistics

Until the 1980s and 1990s, in most Caribbean states sexually violent crime and domestic violence cases (the latter was not yet recognized as a crime) were directed to the country's penal system, to which few victims had recourse. These states can now rely on the UN CEDAW (1979), the Inter-American Convention of Belém do Para (1994) and on the program of action adopted in Beijing in 1995. Since 1991, most states in the region have enacted civil legislation on domestic and sexual violence, inspired by the models prepared by the CARICOM Secretariat, to supplement their penal provisions and protection orders aimed at preventing recidivism. Observers note, however, the minimal effectiveness of these measures. Limited, fragmented and unstandardized, the statistical data, however, furnishes a glimpse of the magnitude and frequency of violence against women in the Caribbean.
\end{abstract}

